\title{
Design of Thermoelectric Power Generation Based on Engine Exhaust
} gas

\author{
Haiyan Dai \& Miaolin Li \\ School of Automotive and Transportation Engineering, Guangzhou College of South China University of \\ Technology, Guangzhou 510800, China
}

\begin{abstract}
In order to improve the thermal efficiency and fuel economy of automobile engine, make full use of the waste heat of automobile exhaust for energy saving and emission reduction, a flat-plate type of thermoelectric power generation(TEG) device is designed which makes full use of the car exhaust gas waste heat. Firstly, introduce the basic principle of the TEG; choose the suitable TEG sheet according to the operating characteristics of general gasoline engine. Secondly, establish the device model of exhaust waste heat TEG by CATIA 3D software, choose the install method and quantity of TEG sheet, design the suitable circuit for the system, at the same time, analyze of the basic application of the device. Finally, according to the data analysis and calculation, find the cost and feasibility of the TEG device. The calculation results show that, the fuel saving ratio of the system is $0.03 \mathrm{~L} / \mathrm{h}$ and it can recover the cost in 4 years. So, the TEG device has certain feasibility and extensive application prospect.
\end{abstract}

KEYWORD: exhaust gas, TEG, thermal efficiency, automobile, Fuel economy

\section{INTRODUCTION}

As the development of the national economy and improvement of people's living standard, vehicles have become common transportation tools in daily life. By 2015, the motor vehicle quantities have reached 270 million. Although the government supports and encourages the development and utility of new energy vehicles, vehicles equipped with internal combustion engine are still widely used. Statistics shows that only about $40 \%$ of the total energy that produced from fuel combustion can be used to overcome the various resistances during driving (Weng C C, 2013), and most of the energy is not effectively utilized but released to the atmosphere wastefully. If the waste heat in the engine's exhaust can be recovered and converted into electrical energy by thermoelectric generator, the engine's effective thermal efficiency and fuel economy can be improved to some extent so as to achieve the purpose of energy saving.

\section{THE BASIC PRINCIPLE OF \\ THERMOELECTRIC POWER GENERATION}

The essence of thermoelectric power generation technology is to concerted the temperature difference between the high-temperature heat source and the low-temperature heat source into electrical energy, which is the so called thermoelectric effect. The thermoelectric effects include Seebeck effect,
Peltier effect, Thomson effect, Joule effect and Fourier effect etc, among which the Seebeck effect, discovered by a Germany scientist named T.J.Seebeck in 1821, is applied in the thermoelectric power generation technology. The Seebeck effect reveals that for the PN junction made of p-type semiconductor and n-type semiconductor (p-type: rich in holes, ntype: rich in electrons), and when its $\mathrm{TH}$ joint and TC joint are under different temperature condition, resulting in an temperature difference, there will be a electromotive force between the two sides when the circuit is open; and a current in the circuit when it is close, as shown in Fig. 1 (Tu Xiao-Liang, 2015).

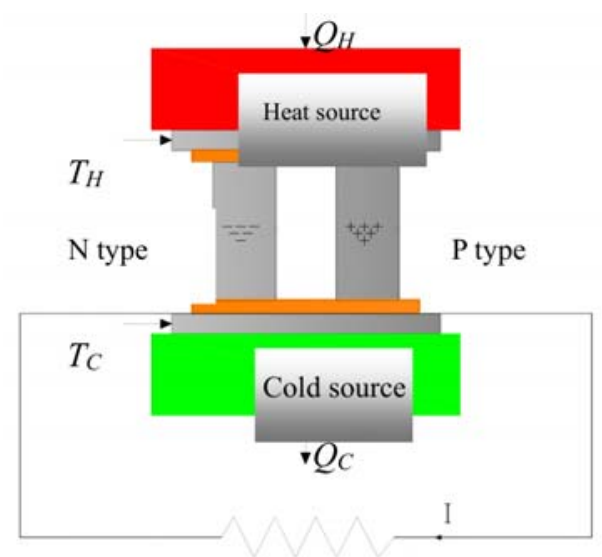

Fig. 1 Seebeck effect 
After several experiments, Seebeck discovered that within a certain range of temperature difference, the electromotive force e of the thermoelectric generation is proportional to the temperature difference between the hot side and cold side(Yao Wu, 2015),

$e=\alpha_{P N}\left(T_{H J}-T_{C J}\right)$

$\alpha_{P N}=\alpha_{P}-\alpha_{N}$

$\alpha_{P N}$ is the semiconductor's relative Seebeck coefficient, $\alpha_{P}, \alpha_{N}$ is the Seebeck coefficient of the semiconductor's $\mathrm{P}, \mathrm{N}$ pole.

Owing to that the electromotive force in single PN junction of the thermoelectric materials is small, the relatively larger current and electromotive force can be achieved by connecting the PN junction in series or in Parallel (Yuan Xiao-hong, 2012).

\section{THE DESIGN OF THERMOELECTRIC POWER GENERATION BASED ON ENGINE EXHAUST GAS}

\subsection{Selection of thermoelectric power module}

The thermoelectric power modules are mainly determined by its materials and structural dimension, and the main supplier includes Amerigon, Ferrotec, Hi-Z in united state; Denso, TOSHIBA, Komatsu in Japan; Thermonamic in domestic market and so on (Xiao Zhe-peng,2014). But thermoelectric power modules are different in properties due to their various structures. Viewing from the perspective of highest heat-resisting temperature, the sectionaltype thermoelectric power modules from the Amerigon Company can reach up to $500^{\circ} \mathrm{C} \sim 700^{\circ} \mathrm{C}$, and this kind of power module can efficiently use the temperature gradient on the hot side surface, resulting an optimal working temperature for each material. Although it has a large generating capacity for single power module, it suffers a high requirement in processing and high price; Komatsu applies BiTe alloy, a middle-low temperature material, in the thermoelectric power module, which has a heat-resisting temperature of $280^{\circ} \mathrm{C}$ (Sun Qiang, 2012); The heatresisting temperature of thermoelectric power modules from the Thermonamic in Jiangxi Province is $330^{\circ} \mathrm{C}$; owing to various limitation and combining with the exhaust gas temperature, the model of TEG127-.4-1.6 with an internal resistance of $3.3 \Omega$ is selected in this design.

\subsection{The Structure Design of TEG Based on Engine Exhaust gas}

Thermoelectric generator is the core of thermoelectric generation system. The traditional thermoelectric generator's structure mainly consists of wasteheat channel, cooling layer, waste heat box layer, power generation group and clamping device for the parts. As most of the thermoelectric power modules are rectangular and inflexible, while most of the vehicles' exhaust conduits are cylindrical, it is impossible for thermoelectric power modules to be installed in the exhaust conduits directly. So, the box structure is applied in the designing of the waste heat box layer, which is used to introduce the exhaust gas as the heat source, namely, the thermoelectric generator is designed to be flat-plate. As shown in Fig. 2, its working components mainly consist of three parts, namely, the heat resource collector, the thermoelectric power module group and the cold source device.

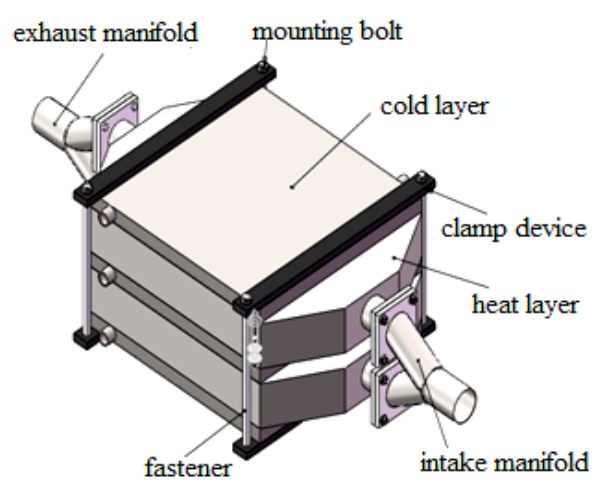

Fig. 2 plate type thermal power generation device

As shown in the Fig. 3, the upper and lower heat layers are acting as the heat collector, referring to the hot side that transfers the heat of the exhaust to the thermoelectric power module group. The cold source device is connected to the engine's cooling system to form the cold side of the thermoelectric power module. Since the automotive cooling system is stable and efficient in heat dissipating, using the cooling system as the cold side can provide a stable temperature difference between the hot side and cold side.

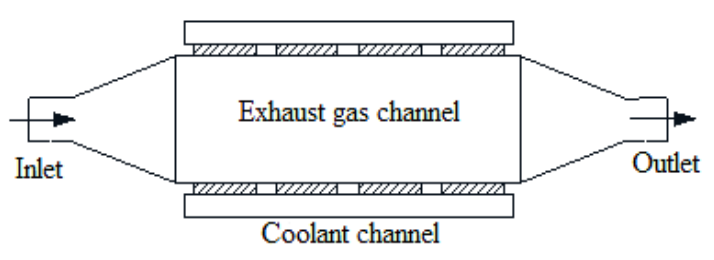

Fig. 3 profile of tail gas thermoelectric generator

\subsection{Material selection for the parts}

(1)heat layer box

The components of the heat layer box are bolted together. Since the exhaust temperature in the inner wall of the heat box is high, and the working condition for the heat box is poor owing to that it is installed at the vehicle's bottom, the material for it must be of heat thermo-stability, anti-thermal expan- 
sively and corrosion resistance. In addition, the heat layer box is also the heat collector, so it is also needed to be equipped with good thermal conductance and stiffness.

By comparison, No.304 stainless steel is selected as the material for heat layer box due to its good corrosion resistance, low temperature strength, mechanical properties and heat thermo-stability; and also due to that it has no hardening behavior after heat treatment.

(2)Cold layer box and intake and exhaust manifolds

The materials for old layer box and intake and exhaust manifolds are basically similar to the selection of materials for heat layer box, so No.304 stainless steel is also material for the cold layer box and intake and exhaust manifolds

(3)Selection of clamping device material

As the internal part of the system is compressed by the clamp device, the material for clamping device needs to be provided with relative high stiffness and toughness, and also a cheap price. After compared with various materials, the grey cast iron is found to be as the suitable material.

\subsection{Connection of thermoelectric power modules}

So far, the voltages for the vehicle battery are $12 \mathrm{~V}$ or $24 \mathrm{~V}$, and for the $12 \mathrm{~V}$ battery, its charging voltage is commonly between $14 \mathrm{~V}-16 \mathrm{~V}$. If the temperature difference between the hot side and cold side of the single thermoelectric power module is $100^{\circ} \mathrm{C}$, then the electromotive force and current will be about $3.0-4.0 \mathrm{~V}$ and $0.15-0.25 \mathrm{~A}$ respectively. So in this design, five thermoelectric power modules are connected in series into rows (generating $15 \mathrm{~V}$ electromotive force), and four rows of modules are connected in parallel (generating 0.8A current). The gap between two power modules is $12 \mathrm{~mm}$ for the arrangement of conductor and the filling of heat insulator.

\subsection{Location of the thermoelectric generation device}

According to the thermoelectric principle, once the thermoelectric power module is given, the voltage generated by single thermoelectric power module is determined by the temperature at the hot side (since the temperature at the cold side is stable due to the cooling system's stable cooling temperature). Therefore, the thermoelectric generation device needs to be mounted as closely as the manifold. But the limited working temperature range for the previously given thermoelectric power module is about $350^{\circ} \mathrm{C}$, so a position of the manifold with suitable temperature and big enough space is selected when mounting the device. Summarizing the above requirements and characteristics, the thermoelectric generation device is located between the muffler and the three way catalyst.

\section{DESIGN OF RELEVANT CIRCUIT}

As the driving cycle changes in real time, the exhaust gas temperature will vary, resulting in a unstable output voltage of the system. In addition, since the output voltage of the thermoelectric generation device is relatively low due to its material and structural limitation, and the voltage supplied for battery and other power consumption equipments is relatively high, so boosting circuit and voltage stabilizing circuit are equipped in this system. Fig. 4 shows a circuit diagram of DC booster, where the Q-1and Q-2 refers to transistors, which are turned on and off alternately to transfer the current to the primary circuit of the transformer. The input of the transformer is square wave, and the required voltage can be obtained by filtering and rectifying the output at B-1.

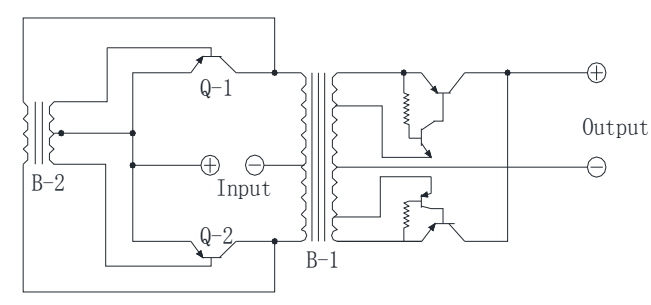

Fig. 4 the circuit diagram of booster

\section{EXPERIMENT DESIGN AND RESULT ANALYSIS}

\subsection{Design of the experiment}

An experiment is designed to test the thermoelectric generation effect in this article. At the hot side, microcomputer heating plate is used to heating the aluminum sandwich heat storage block. At the cold side, the engine's coolant is used to cool the cold side. The load in the circuit is represented by resistance, and the voltage and current are measured by voltmeter and ammeter, the test schematic diagram is shown as Fig. 5.

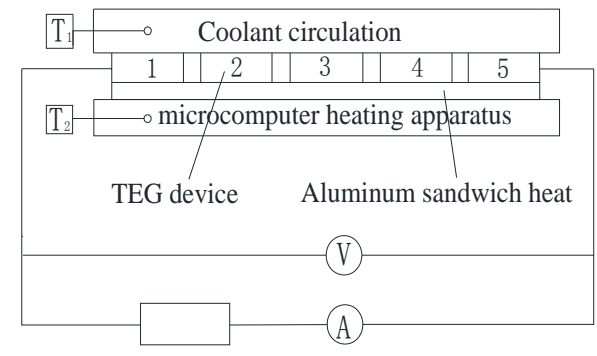

Fig. 5 Test schematic diagram

In order to ensure the reliability and validity of the results, the experiment is needed to be tested 
many times and calculated the average as the final result.

\subsection{Analysis of the test results}

Taking five thermoelectric power modules connected in series relationship as the research object, and the cold resource temperature is controlled at $20^{\circ} \mathrm{C}$, while the hot resource temperature is controlled by the microcomputer heating apparatus. The relationship between the electromotive force caused by thermoelectric generation principle and the temperature difference value can be measured by the test, as shown in Fig. 6.

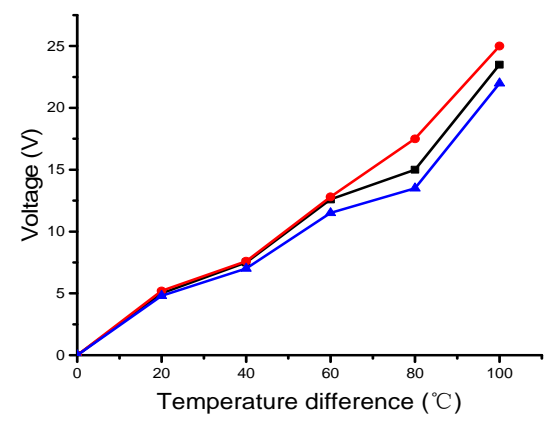

Fig. 6 Relationship between the temperature difference and output voltage

By averaging the test result, it indicates that if the temperature difference value is $100^{\circ} \mathrm{C}$, the electromotive force will be $23.5 \mathrm{~V}$, and the current will be $0.712 \mathrm{~A}$, thus obtaining a power of almost $10 \mathrm{~W}$.

\section{COST AND FEASIBILITY ANALYSIS}

The test results shows that if the temperature difference between the single thermoelectric power module's two ends is $100^{\circ} \mathrm{C}$, it can generate a power of about $2 \mathrm{~W}$. After connecting five modules in one row and connecting 4 rows in parallel relation, the power will be:

$p=2 \times 5 \times 4=40 \mathrm{~W}$

Gasoline density: $\rho=0.725 \mathrm{~g} / \mathrm{ml}$

Fuel consumption of the gasoline engine: about $\frac{40 \times 10^{-3} \times 460}{0.725 \times 10^{3}} L / h=0.03 L / h$

Thus, the fuel saving of the whole system is: $\frac{40 \times 10^{-3} \times 460}{0.725 \times 10^{3}} L / h=0.03 L / h$

The system cost is: the cost for thermoelectric power modules is $20 \times 40=800$; the cost for material and machine processing of heat collector and cooling device is 500 Yuan; the cost for other items is 200 Yuan; and the total cost is 1500 Yuan.

Assuming that the vehicle is driven for six hours every day and 360 days for a year, and for 93\# gasoline price of 5.5 Yuan/L, the cost recovery time will be: $\frac{1500}{5.5 \times 0.03 \times 6 \times 350}=4$ days.

It shows that although it is feasible to use thermoelectric generation principle to realize the wasteheat recovery of car exhaust, the system cost recovery time is up to four years. The major reasons are as follows: firstly, the thermoelectric conversion efficiency in the thermoelectric power module remains relative low; secondly, the heat collection efficiency in the heat collector is needed to be improved to higher level.

According to the calculation, if the generation efficiency of each thermoelectric power module is improved to a power of $5 \mathrm{~W}$, then, the cost recovery time will be reduced to about 1.5 years. Addition, the energy resource shortage is increasingly serious and the oil will be more and more expensive. If the oil price increases to eight Yuan, then the cost recovery time will be further reduced.

\section{SUMMARY}

The automotive exhaust thermoelectric generation technology can efficiently improve the utilization of automotive exhaust waste-heat, resulting in the energy saving and emission reduction purpose and having a promising market prospect and high economy benefit.

Based on the design in this article, further research on thermoelectric material, generation efficiency improvement, the cost reduction and the optimization of the automotive exhaust thermoelectric generation system can lead to further improvement of the vehicles' fuel economy, having a widely application prospect in the car industry.

\section{REFERENCE}

Deng Ya-Dong, Fan Tao, Guo Xun, Ling Kai, Su Chu-qi, Arrangement of TEG Device and Thermoelectric Module [J]. Journal of WUT (Information Management Engineering), 2010, 02:265-267+283.

Jiang Xin-qiang. Research on Semiconductor Thermoelectric Generation System of Vehicle Exhaust Gases[D]. Guangzhou: South China University of Technology, 2010

Love N D.Szybist J P,Sluder C S. Effect of heat exchanger material and fouling on thermoelectric exhaust heat recovery[J].Applied Energy,2012,89(1):322-328.8

N B D,Kim H I,Son J W,et al. The study of a thermoelectric generator with various thermal conditions of exhaust gas from a diesel engine[J].International Journal of Heat \& Mass Transfer,2015,86:667-680.

Stevens R J,Weinstein S J,Koppula K.Theoretical limits of thermoelectric power generation from exhaust gases[J].Applied Energy,2014,133(6):80-88.

Sun Qiang. Automobile Waste Heat Thermoelectric Generator Flow Analysis and Optimization Based on Computational Fluid Dynamics [D]. Wuhan :Wuhan University of Technology, 2012.) 
Tu Xiao-Liang, Ni Ji-min, Shi Xiu-yong. Research on Thermoelectric Power Generation Technology Utilizing Automobile Engine Exhaust Heat[J].Automotive technology, 2015,04:22-25.

Wang Y,Dai C,Wang S. Theoretical analysis of a thermoelectric generator using exhaust gas of vehicles as heat source [J]. Applied Energy,2013,112(4):1171-1180.

Weng C C,Huang M J. A simulation study of automotive waste heat recovery using a thermoelectric power generator [J]. International Journal of Thermal Sciences,2013, 71(3):302309.

$\mathrm{Xu}$ Zhong-lun. Discussion on the formula of fuel consumption rate conversion of gasoline engine[ $\mathrm{J}]$.automotive technology, 1980, 01:9-16.

Xu Li-zhen, Li Yan, Yang zhi, Chen Chang-he. Experimental study of the thermoelectric power generation from automobile exhaust [J]. Journal of Tsinghua University (Sci Tech), 2010, 02:287-289+294.

Xiao Zhe-peng, Wu Wen-ji, Feng Xia. Design and Fabrication Power Generation for Waste Heat Recovery [J]. Mechanical design and manufacture, 2014, 10:259-261.

Yao Wu, Xia Qiang, Zuo Jun-qing. Cathodic Protection of Reinforcing Steel in Simulated Concrete Pore Solution Based on Thermoelectric Generation [J]. Journal of Building Materials, 2015, 01:76-81.

Yuan Xiao-hong. Research on Thermoelectric Technology of Thermoelectric Generation for Automobile Engine Waste Heat [D], Wuhan :Wuhan University of Technology, 2012 